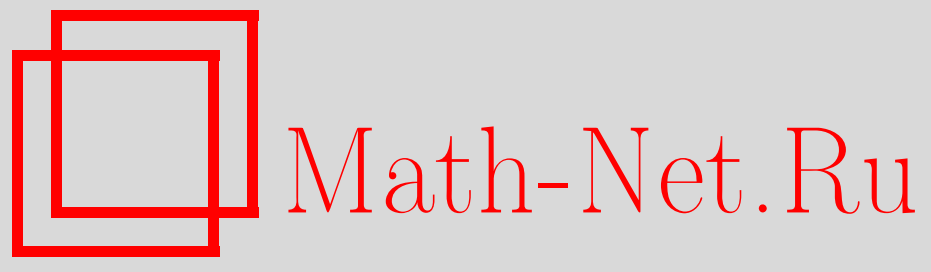

Е. Рафиков, Об оценивании функции регрессии, Матем. заметки, 2006, том 80, выпуск 5, 797-800

DOI: https://doi.org/10.4213/mzm3093

Использование Общероссийского математического портала Math-Net.Ru подразумевает, что вы прочитали и согласны с пользовательским соглашением http://www . mathnet.ru/rus/agreement

Параметры загрузки:

IP : 54.157 .27 .8

26 апреля 2023 г., $14: 42: 27$

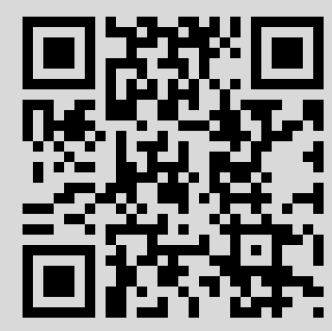




\section{ОБ ОЦЕНИВАНИИ ФУНКЦИИ РЕГРЕССИИ}

\section{Е. Рафиков}

Пусть $X \subset \mathbb{R}^{d}$ и $Y \subset \mathbb{R}$ - борелевские множества, и на их произведении $Z=X \times Y$ определена борелевская вероятностная мера $\rho$. Обозначим условную меру на $Y$ как

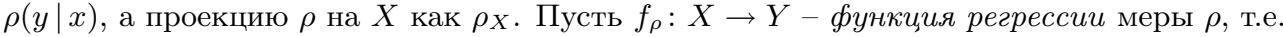

$$
f_{\rho}(x)=\int_{Y} y d \rho(y \mid x)=\mathrm{E}\{y \mid x\}
$$

В статье изучается задача оценивания функции $f_{\rho}(x)$ по конечной $\rho$-выборке $\boldsymbol{z}=\left\{z_{1}\right.$, $\left.\ldots, z_{m}\right\}, z_{i}=\left(x_{i}, y_{i}\right), i=1, \ldots, m$, длины $m$, в случае, когда мера $\rho$ неизвестна. Будем дополнительно предполагать, что $f_{\rho}$ принадлежит некоторому заранее известному функциональному классу $\mathscr{H}$ в пространстве непрерывных ограниченных функций $\mathscr{C}(X)$. Функцию, построенную по выборке $\boldsymbol{z}$ и оценивающую $f_{\rho}(x)$, будем обозначать как $f_{\boldsymbol{z}}: X \rightarrow Y$ и называть функиией-оценщиком.

Естественной характеристикой качества ощенивания функции $f_{\rho}$ представляется скорость убывания вероятностных хвостов (при стремлении $\varepsilon \rightarrow 0$ и $m \rightarrow \infty$ )

$$
\operatorname{Pr}_{\boldsymbol{z} \in Z^{m}}\left\{\left\|f_{\boldsymbol{z}}-f_{\rho}\right\|_{L_{2}\left(X, \rho_{X}\right)}^{2}>\varepsilon\right\},
$$

исследованием которой мы занимаемся ниже.

Многие авторы изучали вопросы качества и оптимальности оценивания $f_{\rho}$ в случае, когда на меру $\rho$ наложены дополнительные условия ограниченности вида $|y|<M \rho$-п.н. для некоторого $M>0$, см. [1]-[3]. Мы покажем, что ряд важных оценок переносится также и на случай неограниченной меры $\rho$. А именно, везде ниже будем предполагать, что $\rho$ удовлетворяет следующим условиям:

$$
\exists C_{1}, C_{2}>0 \quad \text { такие, что } \forall t>0: \quad \operatorname{Pr}_{\rho}\{|y|>t\}<C_{1} \cdot \exp \left\{-C_{2} \cdot t^{4}\right\}, \quad \text { и } \quad f_{\rho}(x) \in \mathscr{H},
$$

где $\mathscr{H} \subset \mathscr{C}(X)$ - компактное множество. В статье приводятся обобщения нескольких утверждений из работы [2].

Пусть $\xi$ - случайная величина со значениями в $\mathbb{R}$. Говорят, что она удовлетворяет свойству субгауссовости, если существуют положительные $C_{1}, C_{2}>0$ такие, что для $t>0$

$$
\operatorname{Pr}\{|\xi|>t\}<C_{1} \cdot \exp \left\{-C_{2} \cdot t^{2}\right\} .
$$

Нам понадобится следующий классический результат (см. [4]).

УтвеРЖДЕнИЕ 1. Пусть $\xi_{1}, \xi_{2}, \ldots, \xi_{m}$ - независимые случайные величины со значениями в $\mathbb{R}$, удовлетворяющие свойству субгауссовости с одинаковыми параметрами $C_{1}, C_{2}>0$. Тогда существует постоянная $C=C\left(C_{1}, C_{2}\right)>0$ такая, что для всех $t>0$ выполняется неравенство

$$
\operatorname{Pr}\left\{\xi_{1}+\xi_{2}+\cdots+\xi_{m}>t\right\} \leqslant e^{-C t^{2} / m} .
$$

Для функции $f: X \rightarrow Y$ из $L_{2}\left(X, \rho_{X}\right)$ назовем ее ошибкой выражение

$$
\mathscr{E}(f):=\mathscr{E}_{\rho}(f):=\int_{Z}(f(x)-y)^{2} d \rho .
$$

Тогда можно проверить, что

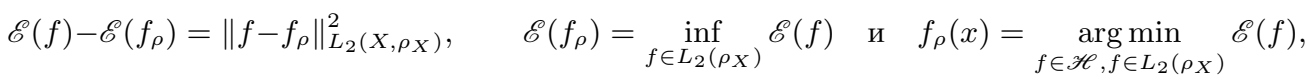

(C) Е. РАфиков, 2006 
а значит для оценщика $f_{\boldsymbol{z}} \in L_{2}\left(X, \rho_{X}\right)$ интересующие нас вероятностные хвосты (1) можно также записать в виде

$$
\operatorname{Pr}_{\boldsymbol{z} \in Z^{m}}\left\{\left\|f_{\boldsymbol{z}}-f_{\rho}\right\|_{L_{2}\left(X, \rho_{X}\right)}^{2}>\varepsilon\right\}=\operatorname{Pr}_{\boldsymbol{z} \in Z^{m}}\left\{\mathscr{E}(f)-\mathscr{E}\left(f_{\rho}\right)>\varepsilon\right\} .
$$

Обозначим эмпирическую ошибку как

$$
\mathscr{E}_{\boldsymbol{z}}(f)=\frac{1}{m} \sum_{i=1}^{m}\left(f\left(x_{i}\right)-y_{i}\right)^{2},
$$

и определим дефект функции $f: X \rightarrow Y$ следующим образом:

$$
L_{\boldsymbol{z}}(f):=L_{\boldsymbol{z}, \rho}(f):=\mathscr{E}(f)-\mathscr{E}_{\boldsymbol{z}}(f) ; \quad \boldsymbol{z}=\left(z_{1}, \ldots, z_{m}\right), \quad z_{i}=\left(x_{i}, y_{i}\right) .
$$

Везде ниже будем считать, что $\mathscr{H} \subset \mathscr{C}(X)$ - компактное подмножество.

Лемма 1. Пусть функиия $f \in \mathscr{H}$ имеет конечную ошибку $\mathscr{E}(f)$. Тогда для некоторой константы $C=C(\mathscr{H}, \rho)>0$ и для всех $\varepsilon>0$ имеет место оценка

$$
\operatorname{Pr}_{\boldsymbol{z} \in Z^{m}}\left\{L_{\boldsymbol{z}}(f)>\varepsilon\right\} \leqslant 2 \exp \left\{-C m \varepsilon^{2}\right\} .
$$

ДоказАтельство. Легко видеть, что случайные величины $\xi_{i}(f)=\left(y_{i}-f\left(x_{i}\right)\right)^{2}-$ $\mathrm{E}_{\rho}\left\{(y-f(x))^{2}\right\}$ независимы. Из условия (2) следует, что они также и субгауссовы с некоторыми одинаковыми параметрами $C_{1}=C_{1}(\mathscr{H}, \rho)$ и $C_{2}=C_{2}(\mathscr{H}, \rho)$. Непосредственное применение утверждения 1 дает нам необходимую оценку.

Обозначим через $N(\mathscr{H}, \varepsilon)$ число покрытий для $\mathscr{H}$ в пространстве $\mathscr{C}(X)$, т.е. наименьшее число шаров радиуса $\varepsilon$ в $\mathscr{C}(X)$ с центрами в $\mathscr{H}$, необходимых для покрытия $\mathscr{H}$. Справедлива

Лемма 2. Существуют константы $C_{j}(\mathscr{H}, \rho)>0, j=1,2$, такие, что для $\varepsilon>0$ выполняется

$$
\operatorname{Pr}_{\boldsymbol{z} \in Z^{m}}\left\{\sup _{f \in \mathscr{H}}\left|L_{\boldsymbol{z}}(f)\right|>\varepsilon\right\} \leqslant 2 N\left(\mathscr{H}, \frac{\varepsilon}{C_{1}(\mathscr{H}, \rho)}\right) \cdot \exp \left\{-C_{2}(\mathscr{H}, \rho) \cdot m \varepsilon^{2}\right\} .
$$

ДокАзАтельство. Числа $c_{k}(\mathscr{H}, \rho)$ ниже обозначают некоторые положительные постоянные. Пусть $f_{1}, f_{2} \in \mathscr{H}$ - произвольные функции. Заметим, что

$$
\left|L_{\boldsymbol{z}}\left(f_{1}\right)-L_{\boldsymbol{z}}\left(f_{1}\right)\right| \leqslant\left|\mathscr{E}\left(f_{1}\right)-\mathscr{E}\left(f_{2}\right)\right|+\left|\mathscr{E}_{z}\left(f_{1}\right)-\mathscr{E}_{z}\left(f_{2}\right)\right| .
$$

Оценим оба слагаемых в правой части по отдельности. Так как

$$
\left(f_{1}(x)-y\right)^{2}-\left(f_{2}(x)-y\right)^{2}=\left(f_{1}(x)-f_{2}(x)\right)\left(f_{1}(x)+f_{2}(x)-2 y\right),
$$

получаем

$$
\begin{aligned}
\left|\mathscr{E}_{(}\left(f_{1}\right)-\mathscr{E}\left(f_{2}\right)\right| & =\left|\int_{Z}\left(f_{1}(x)-f_{2}(x)\right)\left(f_{1}(x)+f_{2}(x)-2 y\right) d \rho\right| \\
& \leqslant\left\|f_{1}-f_{2}\right\|_{\infty} \int_{Z}\left|\left(f_{1}(x)-y\right)+\left(f_{2}(x)-y\right)\right| d \rho \leqslant c_{1}(\mathscr{H}, \rho) \cdot\left\|f_{1}-f_{2}\right\|_{\infty}, \\
\left|\mathscr{E}_{z}\left(f_{1}\right)-\mathscr{E}_{z}\left(f_{2}\right)\right| & =\frac{1}{m}\left|\sum_{i=1}^{m}\left(f_{1}\left(x_{i}\right)-f_{2}\left(x_{i}\right)\right)\left(f_{1}\left(x_{i}\right)+f_{2}\left(x_{i}\right)-2 y_{i}\right)\right| \\
& \leqslant\left\|f_{1}-f_{2}\right\|_{\infty} \frac{1}{m} \sum_{i=1}^{m}\left|\left(f_{1}\left(x_{i}\right)-y_{i}\right)+\left(f_{2}\left(x_{i}\right)-y_{i}\right)\right| .
\end{aligned}
$$


Из условия (2) следует, что случайные величины $\xi_{i}(f)=\left|\left(f_{1}\left(x_{i}\right)-y_{i}\right)+\left(f_{2}\left(x_{i}\right)-y_{i}\right)\right|$ независимы и субгауссовы с некоторыми параметрами $C_{j}=C_{j}(\mathscr{H}, \rho), j=1,2$. В силу утверждения 1 имеем

$$
\operatorname{Pr}_{\boldsymbol{z} \in Z^{m}}\left\{\left|\mathscr{E}_{z}\left(f_{1}\right)-\mathscr{E}_{z}\left(f_{2}\right)\right|>\left\|f_{1}-f_{2}\right\|_{\infty} \cdot t\right\} \leqslant e^{-c_{2}(\mathscr{H}, \rho) \cdot m t^{2}},
$$

откуда для любого $t>0$ получаем неравенство

$$
\operatorname{Pr}_{\boldsymbol{z} \in Z^{m}}\left\{\left|L_{\boldsymbol{z}}\left(f_{1}\right)-L_{\boldsymbol{z}}\left(f_{2}\right)\right|>c_{3}(\mathscr{H}, \rho) \cdot\left(\left\|f_{1}-f_{2}\right\|_{\infty}+t\right)\right\} \leqslant e^{-c_{2}(\mathscr{H}, \rho) \cdot m t^{2}} .
$$

Рассмотрим $f_{1}, \ldots, f_{N}-\varepsilon /\left(4 c_{3}(\mathscr{H}, \rho)\right)$-сеть для $\mathscr{H}$, где $N=N\left(\mathscr{H}, \varepsilon /\left(4 c_{3}(\mathscr{H}, \rho)\right)\right)$, т.е. для любой функции $f \in \mathscr{H}$ можно найти некоторый $f_{j}$ такой, что $\left\|f-f_{j}\right\|_{\infty} \leqslant \varepsilon /\left(4 c_{3}(\mathscr{H}, \rho)\right)$. Из неравенства выше получаем, что существует некоторая универсальная постоянная $c_{4}(\mathscr{H}, \rho)$ такая, что верно

$$
\operatorname{Pr}_{\boldsymbol{z} \in Z^{m}}\left\{\left|L_{\boldsymbol{z}}(f)-L_{\boldsymbol{z}}\left(f_{j}\right)\right|>\frac{\varepsilon}{4}+t\right\} \leqslant e^{-c_{4}(\mathscr{H}, \rho) \cdot m t^{2}}
$$

что дает нам для некоторого $c_{5}(\mathscr{H}, \rho)$ (не зависящего от $\left.j\right)$ и для $t=\varepsilon / 2$ неравенство

$$
\operatorname{Pr}_{\boldsymbol{z} \in Z^{m}}\left\{\left|L_{\boldsymbol{z}}(f)-L_{\boldsymbol{z}}\left(f_{j}\right)\right|>\frac{\varepsilon}{2}\right\} \leqslant e^{-c_{5}(\mathscr{H}, \rho) \cdot m \varepsilon^{2}} .
$$

Для завершения доказательства осталось скомбинировать эту оценку с результатом леммы 1. Заметим, что если $\sup _{f \in \mathscr{H}}\left|L_{\boldsymbol{z}}(f)\right|>\varepsilon$, то найдется $j \in\{1, \ldots, N\}$, для которого выполняется одно из двух неравенств $\left|L_{\boldsymbol{z}}\left(f_{j}\right)\right|>\varepsilon / 2$ или $\left|L_{\boldsymbol{z}}(f)-L_{\boldsymbol{z}}\left(f_{j}\right)\right|>\varepsilon / 2$. Рассмотрим $N$ событий $A_{j}=\left\{\left|L_{\boldsymbol{z}}\left(f_{j}\right)\right|>\varepsilon / 2\right\}$ и $N$ событий $B_{j}=\left\{\left|L_{\boldsymbol{z}}(f)-L_{\boldsymbol{z}}\left(f_{j}\right)\right|>\varepsilon / 2\right\}$ в пространстве $Z^{m}$, где $j=1, \ldots, N$. Тогда справедливо включение

$$
\left\{\sup _{f \in \mathscr{H}}\left|L_{\boldsymbol{z}}(f)\right|>\varepsilon\right\} \subset \bigcup_{j=1}^{n} A_{j} \cup B_{j}
$$

которое позволяет нам написать вероятностную оценку вида

$$
\begin{aligned}
\operatorname{Pr}_{\boldsymbol{z} \in Z^{m}}\left\{\sup _{f \in \mathscr{H}}\left|L_{\boldsymbol{z}}(f)\right|>\varepsilon\right\} & \leqslant \sum_{j=1}^{N} \operatorname{Pr}_{\boldsymbol{z} \in Z^{m}}\left(A_{j}\right)+\operatorname{Pr}_{\boldsymbol{z} \in Z^{m}}\left(B_{j}\right) \\
& \leqslant 2 N\left(\mathscr{H}, \frac{\varepsilon}{c_{6}(\mathscr{H}, \rho)}\right) \cdot \exp \left\{-c_{7}(\mathscr{H}, \rho) \cdot m \varepsilon^{2}\right\}
\end{aligned}
$$

что и завершает доказательство леммы 2.

Приведем оценки вероятностных хвостов (1) в случае, когда в качестве оценщика $f_{\boldsymbol{z}}$ выбирается функция

$$
f_{\boldsymbol{z}}:=f_{\mathscr{H}, \boldsymbol{z}}:=\underset{f \in \mathscr{H}}{\arg \min } \mathscr{E}_{\boldsymbol{z}}(f)
$$

на которой достигается минимум эмпирической ошибки. Обозначим также $\mathscr{E} \mathscr{H}(f):=$ $\mathscr{E}(f)-\mathscr{E}\left(f_{\rho}\right)$ и $\mathscr{E}_{\mathscr{H}}, \boldsymbol{z}(f):=\mathscr{E}_{\boldsymbol{z}}(f)-\mathscr{E}_{\boldsymbol{z}}\left(f_{\rho}\right)$.

Теорема 1. Пусть $\mathscr{H} \subset \mathscr{C}(X)$ - компактное подмножество и мера $\rho$ удовлетворяет условиям (2). Тогда найдутся положительные константы $C_{1}(\mathscr{H}, \rho), C_{2}(\mathscr{H}, \rho)$ такие, что для всех $\varepsilon>0$ справедливо неравенство

$$
\operatorname{Pr}_{\boldsymbol{z} \in Z^{m}}\left\{\mathscr{E}\left(f_{\mathscr{H}, \boldsymbol{z}}\right)-\mathscr{E}\left(f_{\rho}\right)>\varepsilon\right\} \leqslant 2 N\left(\mathscr{H}, \frac{\varepsilon}{C_{1}(\mathscr{H}, \rho)}\right) \cdot \exp \left\{-C_{2}(\mathscr{H}, \rho) \cdot m \varepsilon^{2}\right\} .
$$


ДокАзАтЕЛЬСтво. Утверждение теоремы следует из цепочки неравенств

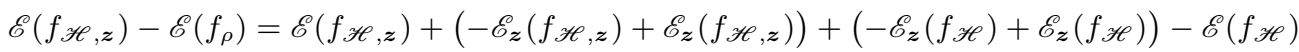

$$
\begin{aligned}
& =\underbrace{\left.\left(\mathscr{E}_{\left(f_{\mathscr{H}}, \boldsymbol{z}\right.}\right)-\mathscr{E}_{\boldsymbol{z}}\left(f_{\mathscr{H}, \boldsymbol{z}}\right)\right)}_{=L_{\boldsymbol{z}}\left(f_{\mathscr{H}, \boldsymbol{z}}\right)}+\underbrace{\left(\mathscr{E}_{\boldsymbol{z}}\left(f_{\mathscr{H}, \boldsymbol{z}}\right)-\mathscr{E}_{\boldsymbol{z}}\left(f_{\mathscr{H}}\right)\right)}_{\leqslant 0}+\underbrace{\left(\mathscr{E}_{\boldsymbol{z}}\left(f_{\mathscr{H}}\right)-\mathscr{E}_{\left.\left(f_{\mathscr{H}}\right)\right)}\right.}_{=-L_{\boldsymbol{z}}\left(f_{\rho}\right)} \\
& \leqslant L_{\boldsymbol{z}}\left(f_{\mathscr{H}, \boldsymbol{z}}\right)-L_{\boldsymbol{z}}\left(f_{\rho}\right)
\end{aligned}
$$

и следующих простых наблюдений:

$$
\begin{aligned}
\operatorname{Pr}_{\boldsymbol{z} \in Z^{m}}\left\{\mathscr{E}\left(f_{\mathscr{H}, \boldsymbol{z}}\right)-\mathscr{E}\left(f_{\rho}\right)>\varepsilon\right\} & \leqslant \operatorname{Pr}_{\boldsymbol{z} \in Z^{m}}\left\{L_{\boldsymbol{z}}\left(f_{\mathscr{H}, \boldsymbol{z}}\right)-L_{\boldsymbol{z}}\left(f_{\rho}\right)>\varepsilon\right\} \\
& \left.\leqslant \operatorname{Pr}_{\boldsymbol{z} \in Z^{m}}\left\{\mid L_{\boldsymbol{z}}\left(f_{\mathscr{H}, \boldsymbol{z}} \mid>\varepsilon\right)\right\}+\operatorname{Pr}_{\boldsymbol{z} \in Z^{m}}\left\{\left|L_{\boldsymbol{z}}\left(f_{\rho}\right)\right|>\varepsilon\right)\right\} \\
& \leqslant 2 \operatorname{Pr}_{\boldsymbol{z} \in Z^{m}}\left\{\sup _{f \in \mathscr{H}}\left|L_{\boldsymbol{z}}(f)\right|>\varepsilon\right\},
\end{aligned}
$$

а также из леммы 2.

Оказывается удобным записать оценки предыдущей теоремы 1 для случая, когда $\mathscr{H}$ характеризуется скоростью убывания своих энтропийных чисел $\varepsilon_{n}(\mathscr{H})$ в пространстве $\mathscr{C}(X)$. Напомним, что

$$
\varepsilon_{n}(\mathscr{H}):=\inf \left\{\varepsilon: \exists f_{1}, \ldots, f_{2^{n}} \in \mathscr{H}: \mathscr{H} \subset \bigcup_{j=1}^{2^{n}}\left(f_{j}+\varepsilon U(\mathscr{C}(X))\right)\right\},
$$

где $U(\mathscr{C}(X))$ - замкнутый единичный шар в $\mathscr{C}(X)$. Имеет место следующая

Теорема 2. Пусть для некоторых $r>0, C>0$ и всех натуральных чисел $n \in \mathbb{N}$ верно $\varepsilon_{n}(\mathscr{H}) \leqslant C n^{-r}$. Тогда существуют положительные константы $C_{1}(r, \rho), C_{2}(r, \rho)>0$ такие, что

$$
\operatorname{Pr}_{\boldsymbol{z} \in Z^{m}}\left\{\mathscr{E}\left(f_{\mathscr{H}, \boldsymbol{z}}\right)-\mathscr{E}\left(f_{\rho}\right) \geqslant \varepsilon\right\} \leqslant e^{-C_{1} \cdot m \varepsilon^{2}}, \quad \text { как толъко } \varepsilon \cdot m^{r /(1+2 r)}>C_{2} .
$$

ДокАЗАтЕльство. Из определения энтропийных чисел следует, что $N(\mathscr{H}, \varepsilon) \leqslant$ $2^{(C / \varepsilon)^{1 / r}}$. Тогда оценка из предыдущей теоремы перепишется как

$$
2 N\left(\mathscr{H}, \frac{\varepsilon}{\widetilde{C}_{1}(\mathscr{H}, \rho)}\right) \cdot \exp \left\{-\widetilde{C}_{2}(\mathscr{H}, \rho) \cdot m \varepsilon^{2}\right\}=\exp \left\{\widetilde{C}_{3}(r, \rho) \cdot \varepsilon^{-1 / r}-\widetilde{C}_{4}(r, \rho) \cdot m \varepsilon^{2}\right\},
$$

где числа $\widetilde{C}_{1}, \widetilde{C}_{2}, \widetilde{C}_{3}, \widetilde{C}_{4}$ положительны. Выберем $\varepsilon \sim m^{\gamma}$ так, чтобы $\varepsilon^{-1 / r}$ и $m \varepsilon^{2}$ имели одинаковые порядки по $m$, т.е. чтобы выполнялось равенство $-\gamma / r=1+2 \gamma$. Тогда, подставляя решение, получаем $\varepsilon=\widetilde{C} \cdot m^{-r /(1+2 r)}$, где $\widetilde{C} \geqslant C_{2}(r, \rho)$ для некоторой постоянной величины $C_{2}(r, \rho)>0$. Легко видеть также, что показатель экспоненты примет вид $\left\{-C_{1} m \varepsilon^{2}\right\}$ для некоторой положительной константы $C_{1}=C_{1}(r, \rho)>0$.

\section{СПИСОК ЦИТИРОВАННОЙ ЛИТЕРАТУРЫ}

[1] L. Györfi, M. Kohler, A. Krzyżak, H. Walk, A Distribution-Free Theory of Nonparametric Regression, Springer Series in Statistics, Springer-Verlag, New York, 2002. [2] F. Cucker, S. Smale, Bull. Amer. Math. Soc., 39 (2001), 1-49. [3] V. Temlyakov, "Approximation in learning theory", IMI Preprints, 5 (2005), 1-43. [4] В. В. Петров, Суммы независимых случайных величин, Наука, М., 1972.

\section{Е. Рафиков}

Поступило

Московский государственный университет 28.06.2006 им. М. В. Ломоносова 